\title{
CERTAIN CLASSES OF MEROMORPHIC FUNCTIONS WITH POSITIVE COEFFICIENTS
}

\author{
NAK EUN CHO AND JI A KIM
}

Abstract. Let $\sum_{p}$ denote the class of functions of the form

$$
f(z)=\frac{a_{-1}}{z}+\sum_{k=1}^{\infty} a_{k} z^{k} \quad\left(a_{k} \geq 0, \quad a_{-1}>0\right)
$$

which are analytic in the annulus $D=\{z|0<| z \mid<1\}$. Let $\Sigma_{p, 1}$ and $\Sigma_{p, 2}$ denote subclasses of $\Sigma_{p}$ satisfying $f\left(z_{0}\right)=\frac{1}{z_{0}}$ and $f^{\prime}\left(z_{0}\right)=-\frac{1}{z_{0}^{2}}\left(-1<z_{0}<1, z_{0} \neq 0\right)$, respectively. Properties of certain subclasses of $\Sigma_{p, 1}$ and $\Sigma_{p, 2}$ are investigated and sharp results are obtained. Also a new characterization for certain subclass of $\Sigma_{p}$ is proved.

\section{Introduction}

Let $\Sigma$ denote the class of functions of the form

$$
f(z)=\frac{a_{-1}}{z}+\sum_{k=1}^{\infty} a_{k} z^{k}\left(a_{-1} \neq 0\right)
$$

which are analytic in the annulus $D=\{z: 0<|z|<1\}$. The Hadamard product or convolution of two functions $f, g$ in $\Sigma$ will denoted by $f * g$. Let

$$
D^{n} f(z)=\frac{1}{z(1-z)^{n+1}} * f(z)\left(z \in D, n \in N_{0}=\{0,1,2, \cdots\}\right) .
$$

Uralegaddi and Ganigi [3] observed that

$$
D^{n} f(z)=\frac{1}{z}\left(\frac{z^{n+1} f(z)}{n !}\right)^{(n)}\left(z \in D, n \in N_{0}\right) .
$$

Received March 16, 1993, revised December 28, 1993.

1991 Mathematics Subject Classification. 30C45.

Key words and phrases. Hadamard product, extreme points, radius of convexity, closure theorems. 
Also, we note that $D^{0} f=f$.

Let $\Sigma_{p}$ be the subclass of $\Sigma$ consisting functions of the form

$$
f(z)=\frac{a_{-1}}{z}+\sum_{k=1}^{\infty} a_{k} z^{k}\left(a_{k} \geq 0, a_{-1}>0\right)
$$

Let $\sum_{p}(n, A, B, \alpha)$ denote the class of functions $f \in \sum_{p}$ such that

$$
\begin{gathered}
\frac{z\left(D^{n} f(z)\right)^{\prime}}{D^{n} f(z)}=-\frac{1+[B+(A-B)(1-\alpha)] w(z)}{1+B w(z)} \\
\left(-1 \leq A<B \leq 1, A+B \geq 0,0 \leq \alpha<1, n \in N_{0}\right),
\end{gathered}
$$

where $z \in U=\{z:|z|<1\}$ and $w \in H=\{w$ analytic in $U, w(0)=0$ and $|w(z)|<$ $1, z \in U\}$.

For a, given real number $z_{0}\left(-1<z_{0}<1, z_{0} \neq 0\right)$, let $\Sigma_{p, 1}$ and $\Sigma_{p, 2}$ be the subclasses of $\Sigma_{p}$ satisfying $f\left(z_{0}\right)=\frac{1}{z_{0}}$ and $f^{\prime}\left(z_{0}\right)=-\frac{1}{z_{0}^{2}}$, respectively. follows:

Let $\Sigma_{p, 1}\left(z_{0}, n, A, B, \alpha\right)$ and $\Sigma_{p, 2}\left(z_{0}, n, A, B, \alpha\right)$ be the subclasses of $\Sigma_{p}$ defined as

$$
\Sigma_{p, i}\left(z_{0}, n, A, B, \alpha\right)=\Sigma_{p}(n, A, B, \alpha) \cap \Sigma_{p, i}(i=1,2) .
$$

In this paper we obtain necessary and sufficient conditions for functions to be in $\Sigma_{p}(n, A, B, \alpha)$ and $\Sigma_{p, i}\left(z_{0}, n, A, B, \alpha\right)(i=1,2)$. We determine extreme points and radius of conexity for the classes $\Sigma_{p, i}\left(z_{0}, n, A, B, \alpha\right)(i=1,2)$. Also closure theorems are proved for these subclasses. Further a new characterization theorem is proved for the class $\Sigma_{p}(n, A, B, \alpha)$. Techniques used are similar to those of Silverman [4].

Remarks. (1). Taking $n=0, \alpha=0$ and $a_{-1}=1$ in the class $\sum_{p}(n, A, B, \alpha)$, we can obtain the results studied by Cho [2].

(2). Taking $n=0, A=-\beta, B=\beta(0<\beta \leq 1)$ and $a_{-1}=1$ in the class $\Sigma_{p}(n, A, B, \alpha)$, we can get the results studied by Mogra, Reddy and Juneja [1].

\section{The Main Resullts}

We now introduce the following notations for brevity:

$$
\begin{aligned}
& D_{k}=(n+k+1) ![(k+1)(B+1)+(A-B)(1-\alpha)], \\
& E_{k}=n !(k+1) !(B-A)(1-\alpha), F_{k}=D_{k}+E_{k} z_{0}^{k+1} .
\end{aligned}
$$

Theorem 2.1. A function $f \in \Sigma_{p}$ is in $\Sigma_{p}(n, A, B, \alpha)$ if and only if

$$
\sum_{k=1}^{\infty} \frac{D_{k} a_{k}}{E_{k}} \leq a_{-1} .
$$


Proof. Suppose $f \in \Sigma_{p}(n, A, B, \alpha)$. Then

$$
\begin{gathered}
\frac{z\left(D^{n} f(z)\right)^{\prime}}{D^{n} f(z)}=-\frac{1+[B+(A-B)(1-\alpha)] w(z)}{1+B w(z)} \\
(-1 \leq A<B \leq 1, A+B \geq 0, w(z) \in H, 0 \leq \alpha<1, z \in U) .
\end{gathered}
$$

From (2.2), we get

$$
w(z)=-\frac{z\left(D^{n} f(z)\right)^{\prime}+D^{n} f(z)}{B z\left(D^{n} f(z)\right)^{\prime}+[B+(A-B)(1-\alpha)] D^{n} f(z)}
$$

and $|w(z)|<1$ implies

$$
|w(z)|=\left|\frac{\sum_{k=1}^{\infty} \frac{(n+k+1) !}{n !(k+1) !}(k+1) a_{k} z^{k}}{(B-A)(1-\alpha) \frac{a_{-1}}{z}-\sum_{k=1}^{\infty} \frac{(n+k+1) !}{n !(k+1) !}[(k+1) B+(A-B)(1-\alpha)] a_{k} z^{k}}\right|<1 .
$$

Since $|\operatorname{Re}(z)| \leq|z|$, we have, from (2.4),

$$
\operatorname{Re}\left(\frac{\sum_{k=1}^{\infty} \frac{(n+k+1) !}{n !(k+1) !}(k+1) a_{k} z^{k+1}}{(B-A)(1-\alpha) a_{-1}-\sum_{k=1}^{\infty} \frac{(n+k+1) !}{n !(k+1) !}[(k+1) B+(A-B)(1-\alpha)] a_{k} z^{k+1}}\right)<1 .
$$

We consider real values of $z$ and take $z=r$ with $0 \leq r<1$. Then, for sufficiently small $r$, the denominator of (2.5) is positive and so it is positive for all $r$ with $0 \leq r<1$, since $w(z)$ is analytic for $|z|<1$. Then (2.5) gives

$$
\sum_{k=1}^{\infty} \frac{D_{k} a_{k} r^{k+1}}{E_{k}}<a_{-1}
$$

Letting $r \rightarrow 1$, we get (2.1).

Conversely, suppose $f \in \Sigma_{p}$ and $f$ satisfies (2.1). For $|z|=r, 0 \leq r<1,(2.6)$ is implied by (2.1), since $r^{k+1}<1$. So we have

$$
\begin{aligned}
& \left|\sum_{k=1}^{\infty} \frac{(n+k+1) !}{n !(k+1) !}(k+1) a_{k} z^{k+1}\right| \leq \sum_{k=1}^{\infty} \frac{(n+k+1) !}{n !(k+1) !}(k+1) a_{k} r^{k+1} \\
& <(B-A)(1-\alpha) a_{-1}-\sum_{k=1}^{\infty} \frac{(n+k+1) ![(k+1) B+(A-B)(1-\alpha)]}{n !(k+1) !} a_{k} r^{k+1} \\
& \leq\left|(B-A)(1-\alpha) a_{-1}-\sum_{k=1}^{\infty} \frac{(n+k+1) ![(k+1) B+(A-B)(1-\alpha)]}{n !(k+1) !} a_{k} z^{k+1}\right|,
\end{aligned}
$$

which gives (2.4) and hence follows that

$$
\frac{z\left(D^{n} f(z)\right)^{\prime}}{D^{n} f(z)}=-\frac{1+[B+(A-B)(1-\alpha)] w(z)}{1+B w(z)}
$$




$$
(-1 \leq A<B \leq 1, A+B \geq 0, w \in H, 0 \leq \alpha<1, z \in U) .
$$

That is, $f \in \Sigma_{p}(n, A, B, \alpha)$.

Theorem 2.2. A function $f \in \Sigma_{p, 1}$ is in $\Sigma_{p, 1}\left(z_{0}, n, A, B, \alpha\right)$ if and only if

$$
\sum_{k=1}^{\infty} \frac{F_{k} a_{k}}{E_{k}} \leq 1
$$

Proof. Let $f \in \Sigma_{p, 1}\left(z_{0}, n, A, B, \alpha\right)$. Then for fixed $z_{0}\left(-1<z_{0}<1, z_{0} \neq 0\right)$, $f\left(z_{0}\right)=\frac{a_{-1}}{z_{0}}+\sum_{k=1}^{\infty} a_{k} z_{0}^{k}$. Since $f\left(z_{0}\right)=\frac{1}{z_{0}}$, we have $a_{-1}=1-\sum_{k=1}^{\infty} a_{k} z_{0}^{k+1}$. Since $f \in \Sigma_{p, 1}\left(z_{0}, n, A, B, \alpha\right), f \in \Sigma_{p}(n, A, B, \alpha)$ and so from Theorem 2.1 and the relation $a_{-1}=1-\sum_{k=1}^{\infty} a_{k} z_{0}^{k+1}$, we get (2.9).

Conversely, let $f \in \Sigma_{p, 1}$ and let (2.9) be satisfied. Since $f\left(z_{0}\right)=\frac{1}{z_{0}}$, we get $\sum_{k=1}^{\infty}$ $a_{k} z_{0}^{k+1}=1-a_{-1}$. Substituting for $\left(1-a_{-1}\right)$ in (2.9), we get (2.1). By Theorem 2.1, we have $f \in \Sigma_{p}(n, A, B, \alpha)$ and hence $f \in \Sigma_{p, 1}\left(z_{0}, n, A, B, \alpha\right)$.

Corollary 2.3. If $f \in \Sigma_{p, 1}\left(z_{0}, n, A, B, \alpha\right)$, then

$$
a_{k} \leq \frac{E_{k}}{F_{k}}\left(k \in N_{0}-\{0\}\right)
$$

with equality for the function

$$
f(z)=\frac{D_{k}+E_{k} z^{k+1}}{z F_{k}}\left(k \in N_{0}-\{0\}\right)
$$

Theorem 2.4. A function $f \in \Sigma_{p, 2}$ is in $\Sigma_{p, 2}\left(z_{0}, n, A, B, \alpha\right)$ if and only if

$$
\sum_{k=1}^{\infty} \frac{\left(D_{k}-k E_{k} z_{0}^{k+1}\right)}{E_{k}} a_{k} \leq 1
$$

Proof. Suppose $f \in \Sigma_{p, 2}\left(z_{0}, n, A, B, \alpha\right)$. Then, for fixed $z_{0}\left(-1<z_{0}<1, z_{0} \neq 0\right)$, $f^{\prime}\left(z_{0}\right)=-\frac{a_{-1}}{z_{0}^{2}}+\sum_{k=1}^{\infty} k a_{k} z_{0}^{k-1}$. Since $f^{\prime}\left(z_{0}\right)=-\frac{1}{z_{0}^{2}}$, we have $a_{-1}=1+\sum_{k=1}^{\infty} k a_{k} z_{0}^{k+1}$. Since $f \in \Sigma_{p, 2}\left(z_{0}, n, A, B, \alpha\right), f \in \Sigma_{p}(n, A, B, \alpha)$ and so Theorem 2.1 holds for $f$. Hence, substituting $a_{-1}=1+\sum_{k=1}^{\infty} k a_{k} z_{0}^{k+1}$ in (2.1), we get (2.12).

Conversely, let $f \in \Sigma_{p, 2}$ and let (2.12) be satisfied. Since $f^{\prime}\left(z_{0}\right)=-\frac{1}{z_{0}^{2}}$, we have $\sum_{k=1}^{\infty} k a_{k} z_{0}^{k+1}=a_{-1}-1$. Substituting the value of $\sum_{k=1}^{\infty} k a_{k} z_{0}^{k+1}$ in (2.12), we get (2.1). From Theorem 2.1, $f \in \Sigma_{p, 2}\left(z_{0}, n, A, B, \alpha\right)$. 


\section{Closure Theorems}

Theorem 3.1. The class $\Sigma_{p, 1}\left(z_{0}, n, A, B, \alpha\right)$ is closed under convex linear combination.

Proof. Let the functions $f_{i}(z)=a_{-1, i} \frac{1}{z}+\sum_{k=1}^{\infty} a_{k, i} z^{k}\left(a_{k, i} \geq 0, a_{-1, i}>0\right)$ be in the class $\Sigma_{p, 1}\left(z_{0}, n, A, B, \alpha\right)$ for $i=1,2, \cdots, m$. We have to show that if the function $h$ is defined by $h(z)=\sum_{i=1}^{m} b_{i} f_{i}(z)\left(b_{i} \geq 0\right)$, where $\sum_{i=1}^{m} b_{i}=1$, then $h$ also belongs to the class $\Sigma_{p, 1}\left(z_{0}, n, A, B, \alpha\right)$. From the definition of $h(z)$, we have

$$
h(z)=\frac{d_{-1}}{z}+\sum_{k=1}^{\infty} d_{k} z^{k},
$$

where $d_{k}=\sum_{i=1}^{m} b_{i} a_{k, i}$. Since $f_{i}(z)$ are in $\Sigma_{p, 1}\left(z_{0}, n, A, B, \alpha\right)$ for $i=1,2, \cdots, m$, we have from Theorem 2.2 ,

$$
\sum_{k=1}^{\infty} \frac{F_{k} a_{k, i}}{E_{k}} \leq 1, i=1,2, \cdots, m
$$

Therefore we have

$$
\begin{aligned}
\sum_{k=1}^{\infty} \frac{F_{k}}{E_{k}}\left(\sum_{i=1}^{m} b_{i} a_{k, i}\right) & =\sum_{i=1}^{m} b_{i}\left(\sum_{k=1}^{\infty} \frac{F_{k}}{E_{k}} a_{k, i}\right) \\
& \leq \sum_{i=1}^{m} b_{i}=1 .
\end{aligned}
$$

This show that the function $h$ belongs to the class $\Sigma_{p, 1}\left(z_{0}, n, A, B, \alpha\right)$ and the theorem is proved.

By the similar method of Theorem 3.1, we can prove the following.

Theorem 3.2. The class $\Sigma_{p, 2}\left(z_{0}, n, A, B, \alpha\right)$ is closed under convex linear combination.

Theorem 3.3. Let $f_{0}(z)=\frac{1}{z}$ and

$$
f_{k}(z)=\frac{D_{k}+E_{k} z^{k+1}}{z F_{k}}(k=1,2, \cdots) .
$$

Then $f \in \Sigma_{p, 1}\left(z_{0}, n, A, B, \alpha\right)$ if and only if it can be expressed in the form $f(z)=$ $\sum_{k=0}^{\infty} \mu_{k} f_{k}(z)$, where each $\mu_{k} \geq 0$ and $\sum_{k=0}^{\infty} \mu_{k}=1$.

Proof. Suppose $f(z)=\sum_{k=0}^{\infty} \mu_{k} f_{k}(z)$, where $\mu_{k} \geq 0$ and $\sum_{k=0}^{\infty} \mu_{k}=1$. Then

$$
f(z)=\mu_{0} f_{0}(z)+\sum_{k=1}^{\infty} \mu_{k} f_{k}(z)
$$




$$
\begin{aligned}
& =\mu_{0} \frac{1}{z}+\sum_{k=1}^{\infty} \mu_{k} \frac{D_{k}+E_{k} z^{k+1}}{z F_{k}} \\
& =\left(\mu_{0}+\sum_{k=1}^{\infty} \frac{D_{k}}{F_{k}} \mu_{k}\right) \frac{1}{z}+\sum_{k=1}^{\infty} \frac{E_{k}}{F_{k}} \mu_{k} z^{k} .
\end{aligned}
$$

Now

$$
\begin{aligned}
\sum_{k=1}^{\infty} \frac{F_{k}}{E_{k}} a_{k} & =\sum_{k=1}^{\infty} \frac{F_{k}}{E_{k}} \frac{E_{k}}{F_{k}} \mu_{k}=\sum_{k=1}^{\infty} \mu_{k} \\
& =1-\mu_{0} \leq 1 .
\end{aligned}
$$

Also by definition, we have $f_{k}\left(z_{0}\right)=\frac{1}{z_{0}}$. Therefore

$$
f\left(z_{0}\right)=\sum_{k=0}^{\infty} \mu_{k} f_{k}\left(z_{0}\right)=\sum_{k=0}^{\infty} \mu_{k} \frac{1}{z_{0}}=\frac{1}{z_{0}} .
$$

This implies $f \in \Sigma_{p, 1}$. By Theorem 2.2, $f \in \Sigma_{p, 1}\left(z_{0}, n, A, B, \alpha\right)$.

Conversely, let $f \in \Sigma_{p, 1}\left(z_{0}, n, A, B, \alpha\right)$. Then $a_{-1}=1-\sum_{k=1}^{\infty} a_{k} z_{0}^{k+1}$. Define

$$
\mu_{k}=\frac{F_{k}}{E_{k}} a_{k}, k \geq 1 \text { and } \mu_{0}=1-\sum_{k=1}^{\infty} \mu_{k} .
$$

From Theorem 2.2, we have $\sum_{k=1}^{\infty} \mu_{k} \leq 1$ and so $\mu_{0} \geq 0$.

Now

$$
\begin{aligned}
f(z) & \stackrel{0 R a_{-1}}{z}+\sum_{k=1}^{\infty} a_{k} z^{k}=\mu_{0} \frac{1}{z}+\sum_{k=1}^{\infty} \mu_{k} \frac{1}{z}\left[1+\frac{z^{k+1}-z_{0}^{k+1}}{\mu_{k}} a_{k}\right] \\
& =\mu_{0} \frac{1}{z}+\sum_{k=1}^{\infty} \mu_{k} \frac{D_{k}+E_{k} z^{k+1}}{z F_{k}} \\
& =\mu_{0} f_{0}(z)+\sum_{k=1}^{\infty} \mu_{k} f_{k}(z)=\sum_{k=0}^{\infty} \mu_{k} f_{k}(z) .
\end{aligned}
$$

This completes the proof of the theorem.

In a similar manner, we can prove the following theorem.

Theorem 3.4. Let $f_{0}(z)=\frac{1}{z}$ and

$$
f_{k}(z)=\frac{D_{k}+E_{k} z^{k+1}}{z\left(D_{k}-k E_{k} z_{0}^{k+1}\right)}(k=1,2, \cdots) \text {. }
$$

Then $f \in \Sigma_{p, 2}\left(z_{0}, n, A, B, \alpha\right)$ if and only if it can be expressed in the form $f(z)=$ $\sum_{k=0}^{\infty} \mu_{k} f_{k}(z)$, where each $\mu_{k} \geq 0$ and $\sum_{k=0}^{\infty} \mu_{k}=1$. 
4. The radius of convexity of the classes $\Sigma_{p, i}\left(z_{0}, n, A, B, \alpha\right)(i=1,2)$

Theorem 4.1. Let $f \in \sum_{p}$. If $f \in \sum_{p, 1}\left(z_{0}, n, A, B, \alpha\right)$, then $f$ is meromorphically convex in the disk $|z|<r$, where

$$
r=\inf _{k}\left[\frac{D_{k}}{k(k+2) E_{k}}\right]^{\frac{1}{k+1}} .
$$

The bound is sharp for the function given by (3.4).

Proof. To prove the theorem, it is sufficient to show that

$$
\left|2+\frac{z f^{\prime \prime}(z)}{f^{\prime}(z)}\right| \leq 1
$$

for $|z| \leq r$. Then we have

$$
\begin{aligned}
\left|2+\frac{z f^{\prime \prime}(z)}{f^{\prime}(z)}\right| & =\left|\frac{\sum_{k=1}^{\infty} k(k+1) a_{k} z^{k-1}}{-\frac{a-1}{z^{2}}+\sum_{k=1}^{\infty} k a_{k} z^{k-1}}\right| \\
& \leq \frac{\sum_{k=1}^{\infty} k(k+1) a_{k}|z|^{k+1}}{\left|a_{-1}-\sum_{k=1}^{\infty} k a_{k} z^{k+1}\right|}
\end{aligned}
$$

Consider the values of $z$ for which

$$
|z| \leq \inf _{k}\left[\frac{D_{k}}{k(k+2) E_{k}}\right]^{\frac{1}{k+1}}
$$

that is,

$$
|z|^{k+1} \leq \frac{D_{k}}{k(k+2) E_{k}}
$$

holds. Then

$$
\sum_{k=1}^{\infty} k a_{k}|z|^{k+1} \leq \sum_{k=1}^{\infty} \frac{D_{k}}{(k+2) E_{k}} a_{k}
$$

Now

$$
\sum_{k=1}^{\infty} k a_{k}|z|^{k+1}<a_{-1}
$$

provided

$$
\sum_{k=1}^{\infty} \frac{D_{k} a_{k}}{(k+2) E_{k}}<a_{-1} .
$$

Now if $f \in \Sigma_{p, 1}\left(z_{0}, n, A, B, \alpha\right)$, then

$$
\sum_{k=1}^{\infty} \frac{F_{k} a_{k}}{E_{k}} \leq 1
$$


or

$$
\sum_{k=1}^{\infty} \frac{D_{k}}{E_{k}} a_{k}<1-\sum_{k=1}^{\infty} a_{k} z_{0}^{k+1}=a_{-1}
$$

and since

$$
\sum_{k=1}^{\infty} \frac{D_{k} a_{k}}{(k+2) E_{k}}<\sum_{k=1}^{\infty} \frac{D_{k} a_{k}}{E_{k}}
$$

(4.8) holds. Therefore we can rewrite the denominator of the right hand side of inequality (4.3) for the considered values of $z$, using the fact that

$$
a_{-1}>\sum_{k=1}^{\infty} k a_{k}|z|^{k+1}
$$

Thus

$$
\left|2+\frac{z f^{\prime \prime}(z)}{f^{\prime}(z)}\right| \leq \frac{\sum_{k=1}^{\infty} k(k+1) a_{k}|z|^{k+1}}{a_{-1}-\sum_{k=1}^{\infty} k a_{k}|z|^{k+1}} \leq 1
$$

if

$$
\sum_{k=1}^{\infty} k(k+2) a_{k}|z|^{k+1} \leq a_{-1} .
$$

If $f \in \Sigma_{p, 1}\left(z_{0}, n, A, B, \alpha\right),(4.14)$ is equivalent to

$$
\sum_{k=1}^{\infty}\left[k(k+2)|z|^{k+1}+z_{0}^{k+1}\right] a_{k} \leq 1 .
$$

By Theorem 2.2, $f \in \Sigma_{p, 1}\left(z_{0}, n, A, B, \alpha\right)$ if and only if

$$
\sum_{k=1}^{\infty}\left(\frac{D_{k}}{E_{k}}+z_{0}^{k+1}\right) a_{k} \leq 1
$$

Hence the inequality (4.15) is true if

$$
k(k+2)|z|^{k+1}+z_{0}^{k+1} \leq \frac{D_{k}}{E_{k}}+z_{0}^{k+1}
$$

for all $k$, that is, if

$$
|z| \leq\left[\frac{D_{k}}{k(k+2) E_{k}}\right]^{\frac{1}{k+1}}
$$

for all $k$. This result is sharp for the extremal function

$$
f_{k}(z)=\frac{D_{k}+E_{k} z^{k+1}}{z F_{k}} \quad\left(k \in N_{0}-\{0\}\right) .
$$


Similarly, we can prove the following theorem.

Theorem 4.2. Let $f \in \Sigma_{p}$. If $f \in \Sigma_{p, 2}\left(z_{0}, n, A, B, \alpha\right)$, then $f$ is meromorphically convex in the disk $|z|<r$, where

$$
r=\inf _{k}\left[\frac{D_{k}}{k(k+2) E_{k}}\right]^{\frac{1}{k+1}}
$$

The bound is sharp for the function given by (3.10).

5. New criteria for $\Sigma_{p}(n, A, B, \alpha)$

In order to prove our new characterization theorems, we shall need the followinng lemma due to Mogra, Reddy and Juneja [1].

Lemma 5.1. A function $f(z)$ of the form (1.4) is meromorphically starlike of order $\beta$ if and only if

$$
\sum_{k=1}^{\infty}(k+\beta) a_{k} \leq(1-\beta) a_{-1}(0 \leq \beta<1) .
$$

Theorem 5.2. A function $f(z)$ of the form (1.4) is in the class $\Sigma_{p}(n, A, B, \alpha)$ if and only if $(f * g)(z)$ is meromorphically starlike of order $\beta$ for the function

$$
g(z)=\frac{1}{z}+\sum_{k=1}^{\infty} \frac{(1-\beta) D_{k}}{(k+\beta) E_{k}} z^{k}(0 \leq \beta<1) .
$$

Proof. In view of Lemma 5.1, a function $f(z)$ of the form (1.4) is starlike of order $\beta$ if and only if

$$
\sum_{k=1}^{\infty}\left(\frac{k+\beta}{1-\beta}\right) a_{k} \leq a_{-1}
$$

Thus, using Theorem 2.1, we have

$$
\begin{aligned}
f(z) & \in \Sigma_{p}(n, A, B, \alpha) \Leftrightarrow \sum_{k=1}^{\infty} \frac{D_{k} a_{k}}{E_{k}} \leq a_{-1} \\
& \Leftrightarrow \sum_{k=1}^{\infty}\left(\frac{k+\beta}{1-\beta}\right)\left(\frac{(1-\beta) D_{k}}{(k+\beta) E_{k}}\right) a_{k} \leq a_{-1} \\
& \Leftrightarrow(f * g)(z) \text { is meromorphically starlike of order } \beta .
\end{aligned}
$$

This completes the proof of Theorem 5.2.

\section{Acknowledgement.}

The authors are thankful to the referee for his helpful comments. 


\section{References}

[1] M.L. Mogra, T.R. Reddy and O.P. Juneja, "Meromorphic univalent functions with positive coefficients, "Bull. Austral. Math. Soc., 32(1985), 161-176.

[2] N.E. Cho, "On certain class of meromorphic functions with positive coefficients," Math. Japonica, 34(1989), 901-907.

[3] B.A. Uralegaddi and M.D. Ganigi, "A new criterion for meromorphic convex functions," Tamkang J. Math., 19(1988), 43-48.

[4] H. Silverman, "Extreme points of univalent with two fixed points", Trans. Amer. Math. Soc., 219(1976), 387-395.

Department of Applied Mathematics, National Fisheries University of Pusan, Pusan 608-737, Korea. 